\title{
Arbitrary quantum-state preparation of a harmonic oscillator via optimal control
}

\author{
Katharina Rojan, ${ }^{1}$ Daniel M. Reich, ${ }^{2}$ Igor Dotsenko, ${ }^{3}$ Jean-Michel \\ Raimond, ${ }^{3}$ Christiane P. Koch, ${ }^{2}$ and Giovanna Morigi ${ }^{1}$ \\ ${ }^{1}$ Theoretische Physik, Universität des Saarlandes, D-66123 Saarbrücken, Germany \\ ${ }^{2}$ Theoretische Physik, Universität Kassel, Heinrich-Plett-Str.40, D-34132 Kassel, Germany \\ ${ }^{3}$ Laboratoire Kastler-Brossel, ENS, UPMC-Paris 6, CNRS, \\ collège de France, 24 rue Lhomond, 75005 Paris, France
}

(Dated: July 24, 2018)

\begin{abstract}
The efficient initialization of a quantum system is a prerequisite for quantum technological applications. Here we show that several classes of quantum states of a harmonic oscillator can be efficiently prepared by means of a Jaynes-Cummings interaction with a single two-level system. This is achieved by suitably tailoring external fields which drive the dipole and/or the oscillator. The time-dependent dynamics that leads to the target state is identified by means of Optimal Control Theory (OCT) based on Krotov's method. Infidelities below $10^{-4}$ can be reached for the parameters of the experiment of the ENS group in Paris, where the oscillator is a mode of a high-Q microwave cavity and the dipole is a Rydberg transition of an atom. For this specific situation we analyze the limitations on the fidelity due to parameter fluctuations and identify robust dynamics based on pulses found using ensemble OCT. Our analysis can be extended to quantum-state preparation of continuous-variable systems in other platforms, such as trapped ions and circuit QED.
\end{abstract}

\section{INTRODUCTION}

Control of the quantum dynamics of physical systems lies at the core of quantum technological applications. A key issue is the initialization of nonclassical states which requires a sufficiently high fidelity to permit efficient information processing. In this context, protocols based on quantum optimal control theory [1, 2] have been acquiring increasing relevance. This is due to the flexibility of the approach, that allows for implementing almost arbitrary dynamics [3 $[5]$ over relatively short time scales, see Refs. [6 9] for a few examples.

Here we apply Optimal Control Theory (OCT) based on Krotov's method [10 12] to the efficient preparation of the quantum state of a harmonic oscillator, which interacts for a fixed time with a dipolar transition. The dipolar transition is quasi-resonant with the oscillator frequency and couples to it via a Jaynes-Cummings type of dynamics [13]. This coupling renders the harmonic oscillator controllable. With the proper sequence of pulses, it is possible to perform any desired unitary transformation on the Hilbert space spanned by the dipole states together with the lowest $n$ energy levels of the oscillator [14 16]. Specific implementations of algorithms based on OCT of the Jaynes-Cummings dynamics include quantum state preparation of a trapped ion's center of mass motion [17, 18] and of superconducting circuits [19, 20]. Here, we focus on quantum state preparation of the electromagnetic-field mode of a high-finesse microwave resonator via the interaction with a transition of a Rydberg atom. Our purpose is to theoretically analyze the efficiency of quantum state preparation of a class of nonclassical states, which have been often discussed in the literature. The efficiency of most of the proposed protocols for these states is limited by the onset of decoherence and by the fact that, in some cases, they are based on projective measurements. It is thus desirable to identify generic procedures for identifying deterministic protocols which can be realized over sufficiently fast times to avoid the detrimental effect of decoherence. We address these issues by developing optimal-control based protocols.

Our theoretical analysis makes specific reference to the setup of the experiment in the ENS group in Paris 21, 22]. The elements of the experiment, which are relevant to our study, are schematically illustrated in Fig. 1: A mode of the electromagnetic field is driven by the dipolar transition between two circular-Rydberg states of an atom flying through the resonator. Atom and microwave field mode undergo a textbook realization of the JaynesCummings dynamics [13, 22]. A fixed interaction time is set by selecting the atom's velocity. To this setup we add the control tools, which are classical fields driving the atomic transition and/or the cavity mode. The specific shape of the fields is determined by OCT using Krotov's method. Our target is the realization of specific quantum states of the resonator with fidelities exceeding 0.9999 (and correspondingly infidelities below $10^{-4}$ ).

The time-dependent Hamiltonian we optimize has first been analyzed by Law and Eberly [14] specifically for arbitrary control of the mode of a quantum electromagnetic field. They proposed a procedure based on a sequence of unitary transformations, in which only one coupling element of the Hamiltonian acts at a time. This protocol has the asset to offer physical insight into the dynamics one needs to shape and serves as initial guess to our search. Our procedure, and optimal control in general, goes nevertheless beyond this intuitive procedure, as it simultaneously considers all processes driving the system and thus exploits interference among them. Analysis performed with superconducting circuits demonstrate its better performance over Law-Eberly type of schemes 23].

The article is organized as follows. Section [I introduces the Hamiltonian and the optimization algorithm. 
a)

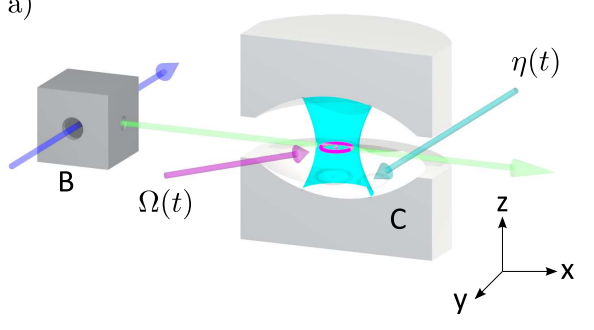

b)

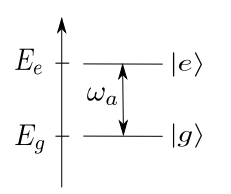

FIG. 1: Scheme of the considered experimental setup. (a) The field of a high-finesse microwave cavity $C$ is prepared in an arbitrary quantum state by means of the interaction with a quasi-resonant dipolar transition of a circular Rydberg atom, schematically shown in (b). The arbitrary superposition of atomic states $|e\rangle$ and $|g\rangle$ is prepared in B. The atom then crosses $\mathrm{C}$ with a fixed velocity, defining the interaction time. The target field state is reached with high fidelity by a suitably tailored time-dependent dynamics identified by means of OCT. The latter delivers the time-dependent form of the electric fields driving the atom, at coupling strength $\Omega(t)$, and the cavity, at strength $\eta(t)$, that efficiently drive the cavity mode into the target state.

Specifically, we discuss the functional which is minimized by means of OCT and the different classes of target states. The results for quantum state preparation are presented in Sec. III] We show how experimental uncertainties and noise can be accounted for in Sec. IV and draw our conclusions in Sec. V]

\section{MODEL}

The physical system we consider is a harmonic oscillator that is coupled to a quasi-resonant two-level transition (dipole) by a Jaynes-Cummings type of interaction. Our objective is to prepare the harmonic oscillator in a specific desired state at the end of a fixed interaction time $\tau$ with the dipole. This is achieved by means of optimized electric fields, which either couple to the atom via a side propagation through the cavity, and/or to the cavity via a diffraction on the mirrors edges or surface defects.

\section{A. Time-dependent Hamiltonian}

Let $\omega_{f}$ be the frequency of the oscillator and $a, a^{\dagger}$ the annihilation and creation operators of an oscillator excitation, with $\left[a, a^{\dagger}\right]=1$. We denote by $|n\rangle$ the numberstate basis of the harmonic oscillator, with $n=0,1,2, \ldots$, such that $a|n\rangle=\sqrt{n}|n-1\rangle$ for $n>1$ and $a|0\rangle=0$. The oscillator couples to a dipolar transition at frequency $\omega_{a}$ with ground state $|g\rangle$ and the excited state $|e\rangle$, as illustrated in Fig. 1(b). The dynamics is generated by the time-dependent Hamiltonian $H(t)$,

$$
H(t)=H_{0}+H_{c}(t) .
$$

Here, $H_{0}$ is the Jaynes-Cummings Hamiltonian, which governs the coupled oscillator-atom dynamics in absence of external drives. It reads

$$
H_{0}=\hbar \omega_{f} a^{\dagger} a+\hbar \omega_{a} \frac{\sigma_{z}}{2}+\hbar \frac{g}{2}\left(a^{\dagger} \sigma+\sigma^{\dagger} a\right),
$$

with $\sigma_{z}$ the Pauli operator for the dipole pseudo-spin and $g$ the vacuum-Rabi frequency, determining the strength of the coupling between oscillator and dipole. For $\omega_{a}=$ $\omega_{f}$ the eigenstates of the Jaynes-Cummings Hamiltonian (22) are the dressed states $| \pm, n\rangle$, which read

$$
| \pm, n\rangle=(|g, n+1\rangle \pm|e, n\rangle) / \sqrt{2},
$$

with eigenvalues $E_{ \pm, n}=\hbar\left(n \omega_{f} \pm g \sqrt{n}\right)$. The coupling between dipole and oscillator gives thus rise to an anharmonic spectrum of excitations of the composite system.

The Hamiltonian $H_{c}(t)$ contains the coupling to the external fields which can induce a time-dependent Stark shift $\Delta(t)$ on the dipolar transition. It can also quasiresonantly drive the atomic transition and cavity mode with time-dependent strengths $\Omega(t)$ and $\eta(t)$, respectively:

$$
\begin{aligned}
H_{c}= & \hbar \Delta(t) \frac{\sigma_{z}}{2}+\hbar \frac{\Omega(t)}{2}\left(e^{-i\left(\phi_{l}(t)+\omega_{l} t\right)} \sigma^{\dagger}+\text { H.c. }\right) \\
& +\hbar \frac{\eta(t)}{2}\left(e^{-i\left(\phi_{p}(t)+\omega_{p} t\right)} a^{\dagger}+\text { H.c. }\right) .
\end{aligned}
$$

The pulses driving cavity and atom have carrier frequencies $\omega_{p}$ and $\omega_{l}$, respectively, with corresponding phases $\phi_{p}$ and $\phi_{l}$. To reduce the numerical effort, we employ a frame that rotates with the oscillator frequency. The total Hamiltonian then reads

$$
\begin{aligned}
H^{\prime}(t)= & \hbar\left(\omega_{a}-\omega_{f}+\Delta(t)\right) \frac{\sigma_{z}}{2}+\hbar \frac{g}{2}\left(a^{\dagger} \sigma+\sigma^{\dagger} a\right) \\
& +\hbar\left(\frac{\tilde{\Omega}(t)}{2} \sigma^{\dagger}+\frac{\tilde{\eta}(t)}{2} a^{\dagger}+\text { H.c. }\right)
\end{aligned}
$$

with complex-valued controls

$$
\begin{aligned}
& \tilde{\Omega}(t)=\Omega(t) e^{-i\left(\omega_{l}-\omega_{f}\right) t} e^{-i \phi_{l}(t)}, \\
& \tilde{\eta}(t)=\eta(t) e^{-i\left(\omega_{p}-\omega_{f}\right) t} e^{-i \phi_{p}(t)} .
\end{aligned}
$$

Equation (5a) accounts for an imposed time-dependent Stark shift of the atomic transition, which are generated by a pulse of amplitude $\vartheta(t)$. We report it for completeness, since the realizations we consider here will not implement this latter kind of pulses ${ }^{1}$.

We seek to identify optimized time dependences of the classical fields which efficiently lead to the preparation

\footnotetext{
1 These pulses can take the form $\Delta(t)=\vartheta^{2}(t) / \delta$ where $\vartheta(t)$ is the slowly-varying amplitude of a third external field coupling state $|g\rangle$ to an auxiliary state $|h\rangle$ at detuning $|\delta| \gg\left|\max _{t}(\vartheta(t))\right|$. In the ENS experiment this can be more simply realized by using the differential Stark effect in a static electric field.
} 
of a target state of the oscillator, denoted by $\left|\Phi_{\text {target }}\right\rangle$, starting from a well defined initial state $|\phi(0)\rangle$ of oscillator and dipole. In particular, we will assume that the oscillator is initially in the ground state, $|\Phi(0)\rangle=|0\rangle$, whereas we take the atom to be in the most convenient state $\left|\Psi_{a}(0)\right\rangle$, depending on the target. The initial state thus reads

$$
|\phi(0)\rangle=\left|\Psi_{a}(0)\right\rangle \otimes|0\rangle
$$

where $\left|\Psi_{a}(t=0)\right\rangle=\alpha|g\rangle+\beta|e\rangle$, with $\alpha=\cos \theta, \beta=$ $e^{i \phi} \sin \theta$ and $\phi, \theta \in \mathbb{R}$. The desired state at time $t=\tau$ has the form

$$
|\phi(\tau)\rangle=\left|\Psi_{a}(\tau)\right\rangle \otimes\left|\Phi_{\text {target }}\right\rangle
$$

where we do not impose any constraint on the atomic state $\left|\Psi_{a}(\tau)\right\rangle$, except for the fact that atom and cavity must be disentagled at time $t=\tau$.

\section{B. Optimal control theory}

In order to determine the classical controls which transfer state (6) into state (7) by the unitary time evolution that is generated by Hamiltonian (5a), we minimize the functional $J$, which is composed of two terms:

$$
J=J_{\tau}+\int_{0}^{\tau} J_{t}[\vartheta(t), \tilde{\Omega}(t), \tilde{\eta}(t)] d t .
$$

The first term on the right-hand side is the final time functional which corresponds to the infidelity, i.e., the difference between unity and the fidelity for obtaining the target state $\left|\Phi_{\text {target }}\right\rangle$,

$$
J_{\tau}=1-\left\langle\Phi_{\text {target }}\left|\operatorname{Tr}_{\mathrm{a}}\left[U(\tau) \rho(0) U^{\dagger}(\tau)\right]\right| \Phi_{\text {target }}\right\rangle .
$$

Here, $U(\tau)=\mathcal{T}\left\{\exp \left(-\mathrm{i} \int_{0}^{\tau} \mathrm{d} t H(t)\right)\right\}$ is the unitary evolution operator generated by $H(t)$ in Eq. (1), with $\mathcal{T}$ being the time-ordering operator, while $\rho(0)$ denotes the initial state of the total system, $\rho(0)=|\phi(0)\rangle\langle\phi(0)|$, and $\operatorname{Tr}_{\mathrm{a}}$ is the partial trace over the dipolar degrees of freedom. The presence of the partial trace indicates that the fidelity for reaching the cavity target state is optimized regardless of the dipole final state. This ideally requires the dipole to be disentagled from the oscillator at time $t=\tau$.
The second term in Eq. (8) is the intermediate-time functional. It explicitly depends on the controls $\vartheta(t)$ (i.e., the field that generates the detuning $\Delta(t)), \tilde{\Omega}(t)$, and $\tilde{\eta}(t)$. A convenient choice corresponds to minimizing the change of the controls with respect to reference fields [5],

$$
\begin{aligned}
J_{t}[\vartheta(t), \tilde{\Omega}(t), \tilde{\eta}(t)]= & \frac{\lambda_{\vartheta}}{S_{\vartheta}(t)}\left[\vartheta(t)-\vartheta_{r e f}(t)\right]^{2} \\
& +\frac{\lambda_{\tilde{\Omega}}}{S_{\tilde{\Omega}}(t)}\left[\tilde{\Omega}(t)-\tilde{\Omega}_{r e f}(t)\right]^{2} \\
& +\frac{\lambda_{\tilde{\eta}}}{S_{\tilde{\eta}}(t)}\left[\tilde{\eta}(t)-\tilde{\eta}_{r e f}(t)\right]^{2} .
\end{aligned}
$$

Here, $S_{\vartheta}(t), S_{\tilde{\Omega}}(t)$ and $S_{\tilde{\eta}}(t)$ are shape functions to ensure a smooth switch on and off of the control fields at times $t=0$ and $t=\tau$. Unless specified otherwise, we take them to be $\sin ^{2}(\pi t / \tau)$, allowing maximum flexibility for shaping $\vartheta(t), \tilde{\Omega}(t)$ and $\tilde{\eta}(t)$. The parameters $\lambda_{\vartheta}, \lambda_{\tilde{\Omega}}$ and $\lambda_{\tilde{\eta}}$ represent weights that govern the step size of the optimization, and $\vartheta_{\text {ref }}(t), \tilde{\Omega}_{\text {ref }}(t)$, and $\tilde{\eta}_{\text {ref }}(t)$ are reference fields. A good choice takes the references fields to be the controls obtained from the previous step of the iterative optimization. This ensures that $J_{t}$ tends to zero as the optimum is approached such that the value of the total functional $J$ close to the optimum is solely determined by the final-time part $J_{\tau}[5]$.

An optimization problem is completely specified in terms of the optimization functional, equations of motion, and coupling to the controls [12]. Based on these ingredients, Krotov's method allows for deriving an optimization algorithm that, in the continuous time limit, guarantees monotonic convergence [10]. It consists in the coupled control equations which need to be solved iteratively. An implementation of Krotov's method is found for example in the spin dynamics software spinach [24, 25]. In our example the linear version of Krotov's method is sufficient for obtaining a monotonically convergent algorithm, since we deal with linear equations of motion, with an intermediate-time functional that is independent of the state of the system, and with a final-time functional that depends only linearly on the state of the system [12]. The update equation for the control, exemplarily given for $\tilde{\Omega}(t)$, reads

$$
\tilde{\Omega}^{(i+1)}(t)=\tilde{\Omega}^{(i)}(t)+\frac{S_{\tilde{\Omega}}(t)}{\lambda_{\tilde{\Omega}}} \mathfrak{I m}\left\{\left\langle\chi^{(i)}(t)\left|\frac{\partial H}{\partial \tilde{\Omega}}\right|_{\tilde{\Omega}^{(i+1)}(t), \varphi^{(i+1)}(t)} \mid \phi^{(i+1)}(t)\right\rangle\right\},
$$

where $i$ denotes the iterative step. The equations for $\tilde{\eta}(t)$ and $\vartheta(t)$ are completely analogous to Eq. (11). Calculating the improved control $\tilde{\Omega}^{(i+1)}(t)$ according to Eq. (11) requires forward propagation of the state of the system $|\phi\rangle$ under the 'new' controls, $\tilde{\Omega}^{(i+1)}(t), \tilde{\eta}^{(i+1)}(t)$, $\vartheta^{(i+1)}(t)$, and backward propagation of the so-called ad- 
joint states $|\chi\rangle$ under the 'old' controls $\tilde{\Omega}^{(i)}(t), \tilde{\eta}^{(i)}(t)$, $\vartheta^{(i)}(t)$. That is, $\left|\phi^{(i+1)}(t)\right\rangle$ and $\left|\chi^{(i)}(t)\right\rangle$ are obtained as the solution of the Schrödinger equations

$\frac{d}{d t}\left|\phi^{(i)}(t)\right\rangle=-\frac{\mathrm{i}}{\hbar} H\left[\vartheta^{(i+1)}(t), \tilde{\Omega}^{(i+1)}(t), \tilde{\eta}^{(i+1)}(t)\right]\left|\phi^{(i)}(t)\right\rangle$,

and

$$
\frac{d}{d t}\left|\chi^{(i)}(t)\right\rangle=-\frac{\mathrm{i}}{\hbar} H\left[\vartheta^{(i)}(t), \tilde{\Omega}^{(i)}(t), \tilde{\eta}^{(i)}(t)\right]\left|\chi^{(i)}(t)\right\rangle .
$$

For our specific choice of optimization functional and Hamiltonian, the equation of motion for the adjoint state $|\chi\rangle$, Eq. (13a), turns simply out to be the standard Schrödinger equation 12. The initial condition for the backward propagation, at final time $\tau$, is given by the derivative of the final-time functional with respect to $\langle\phi|$, evaluated at time $t=\tau$ :

$$
\left|\chi^{(i)}(t=\tau)\right\rangle=-\left.\nabla_{\langle\phi|} J_{\tau}\right|_{\left|\phi^{(i)}(t=\tau)\right\rangle} .
$$

For $J_{\tau}$ in Eq. (8), it becomes

$$
\left|\chi^{(i)}(\tau)\right\rangle=\sum_{a}\left|a, \Phi_{\text {target }}\right\rangle\left\langle a, \Phi_{\text {target }} \mid \phi(\tau)\right\rangle,
$$

where the states $|a\rangle$ correspond to an orthonormal basis of the dipole's Hilbert space. Since Eq. (11) is implicit in $\tilde{\Omega}^{(i+1)}(t)$, the time grids for the states and for the controls are shifted by $\Delta t / 2$ such that $\tilde{\Omega}^{(i+1)}(t+\Delta t / 2)$ is obtained from $\left|\phi^{(i+1)}(t)\right\rangle[5]$. The iteration is started by choosing a guess for each of the controls. The guess fields must be physically sensible choices, otherwise the change in the control in Eq. (11) may be very small and convergence correspondingly slow.

\section{Targets}

Our goal is to determine controls for the preparation of the following classes of states of the harmonic oscillator, namely, (i) Fock states of arbitrary number $n$,

$$
\left|\Phi_{\text {target }}\right\rangle=|n\rangle,
$$

(ii) Fock state superpositions of the form

$$
\left|\Phi_{\text {target }}\right\rangle=\frac{|0\rangle+|n\rangle}{\sqrt{2}}, \quad \text { with } \quad n>1,
$$

and (iii) even cat states such as

$$
\left|\Phi_{\text {target }}\right\rangle=\left|\Phi_{\text {cat }}^{+}\right\rangle=\frac{|\alpha\rangle+|-\alpha\rangle}{\sqrt{2\left(1+e^{-2|\alpha|^{2}}\right)}},
$$

where $|\alpha\rangle=e^{-|\alpha|^{2} / 2} \sum_{n} \alpha^{n} /(\sqrt{n !})|n\rangle$ is a coherent state and $\alpha$ a complex number. The restriction to these classes of states is motivated by the possibility of comparing the efficiency and the dynamics under the optimized controls to previous work which employed protocols based on ingenious intuition of the key elements leading to the desired target states [17, 18, 20, 21, 26 30]. In this way we can acquire a better understanding of the dynamics induced by the optimal control protocol we identify.

There are several physical platforms in which the dynamics discussed here can be implemented. We focus on microwave cavity quantum electrodynamics (QED), where the harmonic oscillator is a mode of a high-finesse microwave resonator and the dipole is a quasi-resonant atomic transition between two Rydberg states. The protocols that have so far been implemented experimentally in microwave cavity QED are based on the interaction of the cavity field with a beam of atoms [21, 31] and in several cases rely on projective measurements. Our purpose is to develop protocols which lead to the deterministic preparation of an arbitrary state of the microwave field with a single atom.

Before presenting our results we recall existing proposals for and implementations of the preparation of Fock states, Fock state superpositions, and Schrödinger cat states. Several proposals for preparing the mode of a resonator in a Fock state can be found in the literature, see e.g. Refs. [21, 26, 30 36]. Fock states have been experimentally realized with the motion of single trapped ions coupled via lasers to an ion internal transition [28], with a high-Q mechanical resonator coupled to superconducting circuits [19, 20], in circuit QED [37], and with the mode of a high-Q cavity coupling to a two-level transition [31, 35, 36, 38 48]. Fock state superpositions, cf. Eq. (15), have been deterministically created with superconducting circuits [19, 20]. The ability of creating such states is required in order to prepare so-called NOON states of two harmonic oscillators [49], which are relevant for quantum metrology. Schrödinger cat states are popular in the literature due to their peculiar nonclassical properties. Remarkable experimental realizations have been reported in microwave cavity QED [50, 51], trapped ions [27, 28, 52], and most recently with superconducting circuits 20].

The efficiency of most of these protocols is limited by the onset of decoherence and by the fact that, in some cases, they are based on projective measurements. It is thus desirable to identify generic procedures for identifying deterministic protocols which can be realized over sufficiently fast times to avoid the detrimental effect of decoherence.

\section{RESULTS}

The time scale $\tau$ of the interaction is fixed in relation to the experimental parameters. In detail, in the setup of the experiment in Paris [21, 22], the transition frequencies are $\omega_{a}=\omega_{f}=2 \pi \times 51 \mathrm{GHz}$, the coupling strength (vacuum Rabi frequency) amounts to $g=2 \pi \times 50 \mathrm{kHz}$. We choose here the interaction times to not exceed $10 \mathrm{~ms}$, which justifies our assumption of unitary time evolution, 
being the atomic transition lifetime $30 \mathrm{~ms}$ and the cavity decay time $0.1 \mathrm{~s}$. In particular, we analyze and compare the efficiency of quantum state preparation for two different time scales: (i) the time scale of the atom-cavity interaction determined by $g$, namely $g \tau \sim 1$, such that $\tau$ ranges in the interval $\tau \sim 10 \mu \mathrm{s}-40 \mu \mathrm{s}$ and (ii) the time scale corresponding to $g \tau \gg 1$, namely, $\tau=10 \mathrm{~ms}$. For such large time scale the dressed states of the coupled atom-cavity system are spectrally resolved up to a precision of the order of $2 \pi \times 0.1 \mathrm{kHz}$.

In order to understand the dynamics induced by the optimized controls and identify the control mechanisms, we analyze the ground and excited state populations of the two-level system,

$$
\rho_{j j}(t)=\operatorname{Tr}\{|\phi(t)\rangle\langle\phi(t)|| j\rangle\langle j|\},
$$

with $j=e, g$, the average photon number in the cavity,

$$
\langle n\rangle_{\phi(t)}=\left\langle\phi(t)\left|a^{\dagger} a\right| \phi(t)\right\rangle
$$

together with its standard deviation,

$$
\Delta n=\sqrt{\left\langle n^{2}\right\rangle_{\phi(t)}-\langle n\rangle_{\phi(t)}^{2}},
$$

and the spectra of the optimized fields $I_{\xi}(\omega)=\left|\xi_{F}(\omega)\right|^{2}$, with

$$
\xi_{F}(\omega)=\frac{1}{2 \pi} \int_{0}^{\tau} \tilde{\xi}(t) e^{-i \omega t} d t
$$

and $\tilde{\xi}=\tilde{\Omega}(t), \tilde{\eta}(t)$.

\section{A. Fock states}

We first consider the preparation of the oscillator in a Fock state, Eq. (14), assuming that at $t=0$ it is in the ground state. The preparation of a number state with $n=1$ follows a very simple dynamics, solely determined by the Jaynes-Cummings Hamiltonian $H_{0}$ in Eq. (2) [14]. In fact, by preparing the atom in the excited state and the cavity in the vacuum, the cavity will end up in a single photon $n=1$ Fock state after the interaction time $\tau=T_{0} / 2$, with $T_{0}=2 \pi / g$. In general, provided that the initial state is $|e, n\rangle$, the Jaynes-Cummings dynamics will naturally let the system evolve to the state $|g, n+1\rangle$ after the time $\tau=(2 m+1) T_{n} / 2$, with $m$ a natural number and

$$
T_{n}=2 \pi /(g \sqrt{n+1}) .
$$

This concept requires control of the initial state and of the interaction time, but no additional field. It is at the basis of several protocols [17, 18, 29, 30] for creating a Fock state starting from the vacuum which is relatively simple to prepare. In detail, the preparation of Fock states with $n>1$ starting from $n=0$ can make use of external fields which perform $\pi$-pulses on the atom after one excitation has been transferred to the resonator. The number of such pulses shall correspond to $n$, such that the interaction time $\tau \geq \tau_{n}$ with $\tau_{n}=\sum_{j=0}^{n} T_{j} / 2$.

On the basis of this knowledge, we choose the initial state of the atom to be $|e\rangle$ and analyze the pulses predicted by OCT for the target state $|n=4\rangle$ and an interaction time $\tau=40 \mu \mathrm{s}$. Note that for the value $g=2 \pi \times 50 \mathrm{kHz}$, the interaction time $\tau$ we choose is of the same order of magnitude as $\tau_{n}$. We assume that only the atom is driven by an external field, i.e., $\tilde{\eta}(t)=0$, $\Delta(t)=0$, since this is relatively simpler to implement in the setup of Ref. [21]. Moreover, amplitude control turns out to be sufficient, i.e., we keep $\phi_{l}(t)=0$, and $\tilde{\Omega}(t)=\Omega(t)$ is real.

Figure 2 displays the temporal (a) and spectral (b) behaviour of the pulse, which leads to a fidelity $\mathcal{F}=$ $1-3 \times 10^{-5}$ for reaching the target state. The driving field consists of a series of pulses, whose effect on the dynamics of the composite system can be inferred from Fig. 2(c) and (d). Figure 2(c) displays the time evolution of the mean intracavity photon number $\langle n\rangle$ and its standard deviation $\Delta n$, cf. Eqs. (18) and (19): The number of photons increases in a stepwise manner, like in protocols based on Refs. [14, 30], in which one photonic excitation at a time is transferred to the cavity mode. Correspondingly, the variance remains limited, and vanishes exactly at $t=\tau$. Figure 2(d) shows the populations of the atom's ground and excited state as a function of time $t$, cf. Eq. (17): They oscillate according to the stepwise increase of the photon number, performing a series of half Rabi cycles. Thus, the protocol obtained numerically essentially follows the analytical intuition: Similar to the proposal of Ref. 14] there is only one active coupling at a time.

At this stage several comments are in order. First, by testing other initial atomic states than $|e\rangle$, it turned out that the choice of $|e\rangle$ leads to better fidelities for preparing Fock states. The second comment concerns the interaction time. Optimization delivers a pulse that maximizes the fidelity for the specified interaction time. Times shorter than $\tau=40 \mu$ s lead to comparable efficiencies, as long as they are longer than $\tau_{n}$. Interaction times shorter than $\tau_{n}$ lead to inefficient dynamics: the fidelity is drastically reduced. We have verified that this is a fundamental limit by allowing for optimization of the additional external fields $\tilde{\eta}(t)$ and $\vartheta(t)$. These optimizations confirmed that $\tau_{n}$ is the minimal time, needed to transfer one excitation to the cavity containing already $n$ excitations. These considerations are quite generic: We have tested them for the preparation of other number states, $n=2$ and $n=4$, yielding the same conclusion. Our analysis thus indicates that $\tau_{n}$ is a good estimate of the quantum speed limit (namely, the minimum time required to transfer a quantum state into another, orthogonal state [53]) for transferring the vacuum to the Fock number state $|n\rangle$. This result, moreover, attests to the ability of OCT to identify the quantum speed limit in cases where an analytical estimate is not possible [7]. 

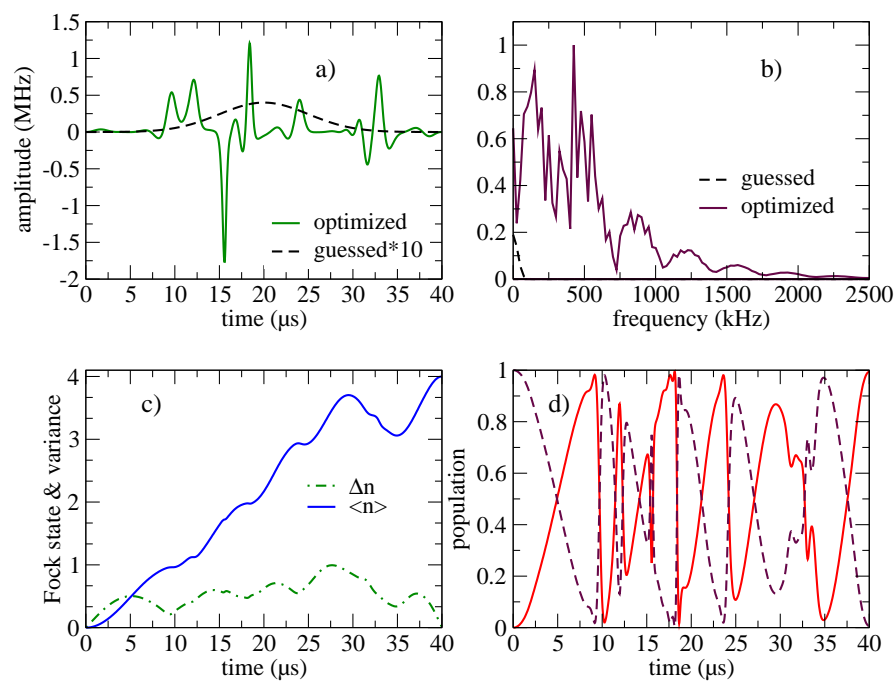

FIG. 2: Preparation of the Fock state $|n=4\rangle$ in $\tau=40 \mu \mathrm{s}$ using OCT: (a) Amplitude of the pulse driving the atom, $\tilde{\Omega}(t)$, as a function of time (in $\mu$ ). The guess pulse (dashed line) is a Gaussian with a maximal amplitude of $E_{0}=40 \mathrm{kHz}$ and $\sigma=$ $5 \mu \mathrm{s}$. (b) Spectrum of the pulse, according to Eq. (20) (since $\tilde{\Omega}(t)=\Omega(t)$ is real, the spectrum is symmetric around 0 , and we show only the positive frequencies). The other subplots show the temporal dependence of (c) mean intracavity-photon number $\langle n\rangle$ (solid line) and its standard deviation $\Delta n$ (dashed line), cf. Eqs. (18) and (19), and (d) population of the atomic states $\rho_{g g}$ (solid line) and $\rho_{e e}$ (dashed line), Eq. (17). The initial state is $|e, 0\rangle$, the other parameters are specified in the text.

We have also verified that it is possible to create other number states with infidelities of the order of $10^{-5}$ using OCT and solely by means of a time-dependent drive on the atom with real amplitude. We note, moreover, that as there are many possible solutions for the shape of the atom pulse, the choice of the guess pulse influences the form of the optimized pulse but not the final infidelity. The preparation of the Fock state $n=1$ is peculiar since in principle it requires no external control, as the JaynesCummings Hamiltonian naturally drives the atom from the excited to the ground state by emitting a photon into the resonator with a half Rabi cycle at the interaction time $\tau_{1}$. Nevertheless, also in this case and for the same interaction time we find time-dependent dynamics based on external pulses, whose maximum fidelity is the same as the one obtained for the Jaynes-Cummings dynamics without external fields. This property is not surprising 54], and reflects a landscape with several possible optimization. We note, in particular, that the solution with no external field can be indeed recovered by OCT, introducing a cost functional that minimizes the integrated pulse energy. Such a cost functional, however, leads to inefficient dynamics for the preparation of Fock states with $n>1$ as well as for the preparation of other classes of states.

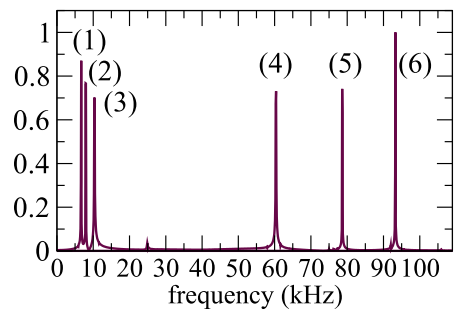

FIG. 3: Preparation of the Fock state $|n=4\rangle$ in $\tau=10 \mathrm{~ms}$ using OCT: Positive-frequency part of the pulse spectrum (in arbitrary units), according to Eq. (20) (the spectrum is symmetric about the resonance frequency since the amplitude is real). The initial state is $|e, 0\rangle$, the other parameters are specified in the text. Differing from the other cases, the shape function used for this optimization is a constant. The numbers in the plot label transitions between dressed states of the atom-cavity system, for details see text.

Even though fast dynamics are in general preferable, the analysis of the optimization results for longer times is instructive. We now focus on the preparation of $|n=4\rangle$ starting from $|e, 0\rangle$ for the interaction time $\tau=10 \mathrm{~ms}$, which is orders of magnitude larger that $\tau_{n}$. This interaction time allows for the spectral resolution of the lowestenergy dressed states of the atom-cavity system. In this regime, a well-defined number of photons can be pumped into the resonator by resonantly driving a dressed state of the Jaynes-Cummings spectrum [35, 36]. Figure 3 displays the spectrum of the optimized pulse which yields an infidelity of $2 \times 10^{-6}$. The amplitude of the optimized pulse is in general three orders of magnitude smaller than that in Fig. 2 and oscillates on the microsecond time scale. The spectrum of the optimized pulse in Fig. 3 exhibits well-defined peaks at the dressed states frequencies. In detail, the peaks which are particularly pronounced correspond to transitions $| \pm, 2\rangle \rightarrow| \pm, 3\rangle$ for (1), $| \pm, 1\rangle \rightarrow| \pm, 2\rangle$ for (2), $| \pm, 0\rangle \rightarrow| \pm, 1\rangle$ for (3), $| \pm, 0\rangle \rightarrow|\mp, 1\rangle$ for (4), $| \pm, 1\rangle \rightarrow|\mp, 2\rangle$ for (5), and $| \pm, 2\rangle \rightarrow|\mp, 3\rangle$ for (6). This shows that, in the limit of a sufficiently long interaction time scale, the protocol tends to address the individual transitions between dressed states, adding sequentially excitations till the target state is reached.

For both $\tau=40 \mu \mathrm{s}$ and $\tau=10 \mathrm{~ms}$, the required numerical effort is very moderate: Figure 2 shows the results of an optimization using 4000 iteration steps, with each iteration taking $5-10 \mathrm{~s}$ on a regular workstation computer. For longer $\tau$ such as in Fig. 3 the CPU time per iteration goes up to $10-20 \mathrm{~s}$ but a much smaller number of iterations, of the order of 100, is required. This indicates that the overall operation time is a resource for control: The control problem becomes simpler as more time is available. Note that the exact number of iterations to reach a certain fidelity threshold depends on the initial guess. 


\section{B. Fock state superpositions}

We now analyze the efficiency of preparing a coherent superposition of Fock states of the form given in Eq. (15) using OCT, and first discuss in detail the efficiency of preparing the superposition

$$
\left|\Phi_{\text {target }}\right\rangle=\frac{|0\rangle+|2\rangle}{\sqrt{2}}
$$

for the interaction time $\tau=20 \mu$ s and solely using pulses driving the atom. Our study thus focuses on optimizing the field described by the control $\tilde{\Omega}(t)$ and shows that complex-valued $\tilde{\Omega}(t)$, that is time-dependent amplitude and phase, lead to higher efficiencies. Therefore, the preparation of Fock-state superpositions requires control over both amplitude and phase of the pulses. This is a logical consequence of the fact that the final state bears a phase information. Figure 4(a) displays the temporal dependence of the pulse, which has been obtained by minimizing the functional to a final infidelity of $2 \times 10^{-5}$. The spectrum is shown in Fig. 4(b): It is broad and asymmetric about the resonance frequency. This feature is due to the complex amplitude of the pulse. Figure 4(c) shows that the intracavity photon number evolves from the vacuum state to the desired target state, which is characterized by a mean photon number $\langle n\rangle=1$ and standard deviation $\Delta n=1$. The atomic level populations in Fig. 4 (d) display some oscillatory behaviour until almost all the population ends up in $|g\rangle$. We note that better efficiencies are found by taking the initial atomic state to be a superposition of ground and excited state: In the case of Fig. 4the initial atomic state is $\left|\Psi_{a}\right\rangle=(|g\rangle+\mathrm{i}|e\rangle) / \sqrt{2}$.

When instead the interaction time is large, $\tau=10 \mathrm{~ms}$, the amplitude of the pulses becomes approximately three orders of magnitude smaller in comparison to the pulses obtained for shorter interaction times. Here, the amplitude changes are carried out on time scales of the order of microseconds. Figure 5 displays the spectrum of the corresponding optimized pulse, which leads to a final infidelity below $10^{-10}$. Since the pulse is real, its form is symmetric about the resonance frequency. The peaks correspond to the frequencies of transitions between dressed states of the atom-cavity system, as follows: (1) corresponds to the transition $| \pm, 1\rangle \rightarrow| \pm, 2\rangle$, (2) to $| \pm, 0\rangle \rightarrow| \pm, 1\rangle$, (4) to $| \pm, 0\rangle \rightarrow|\mp, 1\rangle$ and (5) to $| \pm, 1\rangle \rightarrow|\mp, 2\rangle$, whereas (3) corresponds to the transition from the cavity vacuum state to the dressed states $|\mp, 0\rangle$. The spectrum shows that, for sufficiently long interaction times, quantum-state preparation here is reached by means a pulse which resonantly drives the dressed states transitions leading to the target state.

We finally perform an optimization over the minimum time required to prepare the superposition

$$
\left|\Phi_{\text {target }}\right\rangle_{n}=\frac{|0\rangle+|n\rangle}{\sqrt{2}}
$$
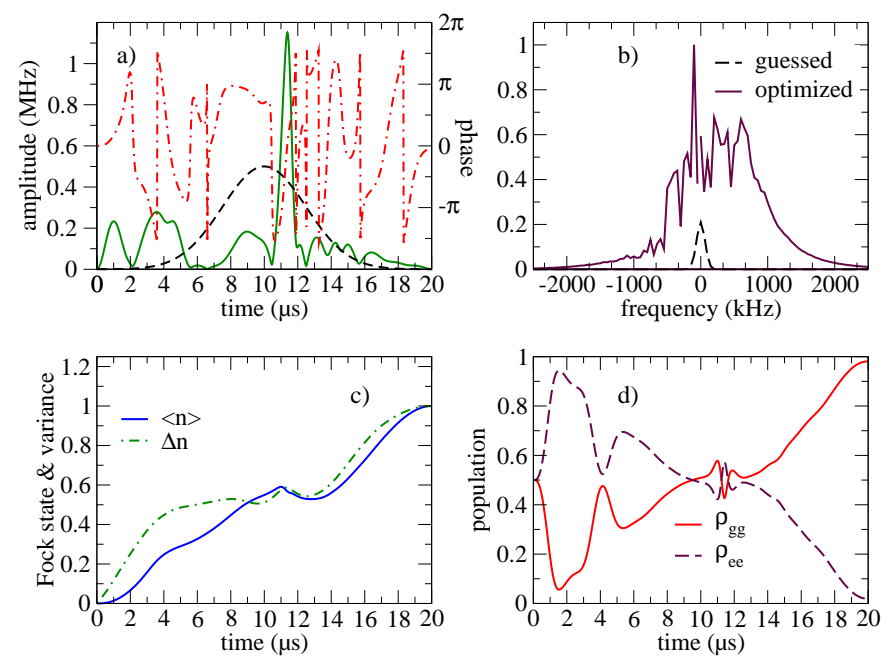

FIG. 4: Preparation of the Fock state superposition $(|0\rangle+$ $|2\rangle) / \sqrt{2}$ in the interaction time $\tau=20 \mu$ s with a pulse driving the atom. (a) Temporal dependence of the pulse amplitude (solid line) and phase (dashed-dotted line). The dashed line shows the guess pulse amplitude (amplified by a factor 10): This is a Gaussian with a maximal amplitude of $E_{0}=50 \mathrm{kHz}$ and $\sigma=2.5 \mu \mathrm{s}$. (b) Spectrum of the pulse, according to Eq. (20). The amplitude is in arbitrary units. The other subplots show the temporal dependence of (c) mean intracavityphoton number $\langle n\rangle$ and standard deviation $\Delta n$, and (d) population of the atomic states $\rho_{g g}$ and $\rho_{e e}$. The initial state is $(|g\rangle+\mathrm{i}|e\rangle) / \sqrt{2} \otimes|0\rangle$.

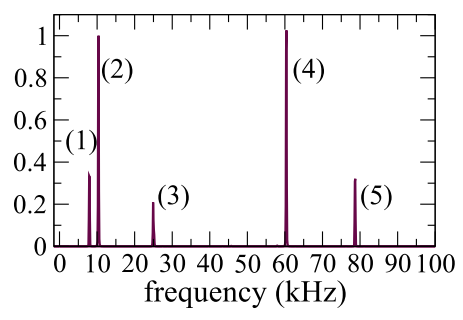

FIG. 5: Preparation of the Fock state superposition $(|0\rangle+$ $|2\rangle) / \sqrt{2}$ for the interaction time $\tau=10 \mathrm{~ms}$. Positivefrequency part of the spectrum of the pulse (in arbitrary units), according to Eq. (20) (since the pulse is real, the spectrum is symmetric about the resonance frequency). The initial state is $(|g\rangle+\mathrm{i}|e\rangle) \otimes|0\rangle / \sqrt{2}$. The shape function used for this optimization is a constant. The numbers label transitions between dressed states, for details see text.

as a function of the Fock-state number $n$. In doing so, we require the infidelity to be below $10^{-4}$. We take the initial state to be

$$
|\phi(t=0)\rangle=\frac{|g\rangle+\mathrm{i}|e\rangle}{\sqrt{2}} \otimes|0\rangle,
$$

and optimize only the atom pump pulse $\tilde{\Omega}(t)$. Figure 6(a) displays the final infidelity $J_{\tau}$ as a function of the interaction time $\tau$ for the preparation of the state 

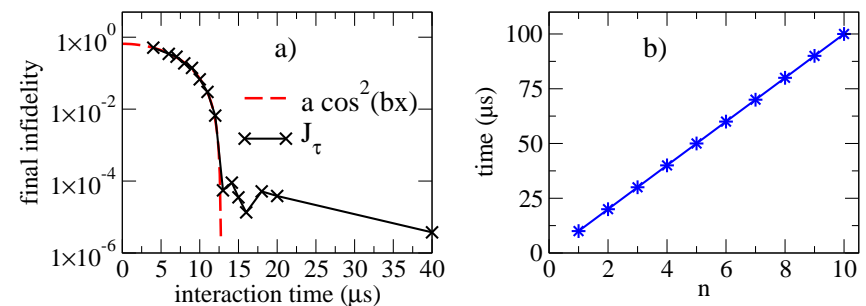

FIG. 6: (a) Final infidelity $J_{\tau}$ as a function of time (in $\mu s$ ) for the preparation of the target state $(|0\rangle+|2\rangle) / \sqrt{2}$. The crosses correspond to the numerical data, the solid line serves as a guide for the eyes, whereas the red dashed line corresponds to the fitted curve $f(x)=a \cos ^{2}(b x)$, with $a=0.25$ and $b=$ 0.66. (b) Minimum time (in $\mu \mathrm{s}$ ) as a function of $n$ required to prepare the target state $(|0\rangle+|n\rangle) / \sqrt{2}$ with infidelity $<10^{-4}$. The initial state is $(|g\rangle+\mathrm{i}|e\rangle) \otimes|0\rangle / \sqrt{2}$, and only the atompump pulse $\tilde{\Omega}(t)$ is employed in Eq. (4).

$(|0\rangle+|2\rangle) / \sqrt{2}$. The behaviour clearly shows that infidelities below $10^{-4}$ are reached for interaction times exceeding $12 \mu \mathrm{s}$. At shorter times, the infidelity decreases with a functional behaviour that can be fitted to the function $f(x)=a \cos ^{2}(b x)$. Analogous behaviour has been reported in other optimization studies [7, 55]. The sharp change in the infidelity allows for the determination of the quantum speed limit, which for this specific state and dynamics lies at about $12 \mu \mathrm{s}$. By means of the same procedure we identify the minimum time required to prepare the state $(|0\rangle+|n\rangle) / \sqrt{2}$ with final infidelity below $10^{-4}$ as a function of $n$. Figure 6(b) shows that the required interaction time scales linearly with $n$. This time exceeds the time found by simple estimates, which should scale as $\sqrt{n}$ for protocols which make use of a third, ancillary state as in Ref. [49]. Here, instead, the scaling of time is found for the evolution constrained to occur between the two states $|g\rangle$ and $|e\rangle$ coupling resonantly to the cavity field.

We note that the preparation of Fock state superpositions is achieved here by solely employing an external pulse that drives the atom. We have also applied optimization of the pulse driving the cavity and that generating a time-dependent dynamical Stark shift on the atom, but found no significant improvement with respect to the case in which only the pulse on the atom was employed. A systematic comparison indeed reveals that the atom pulse leads to the target state in an efficient way, whereas employing solely the pump on the cavity leads to dynamics which are characterized by lower fidelities over time scales of the order of tens of microseconds. The required numerical effort is very similar to the one reported in Section $\amalg$ IIA.

\section{Schrödinger cat states}

In this section we focus on the deterministic preparation of Schrödinger cat states, cf. Eq. (16). They are
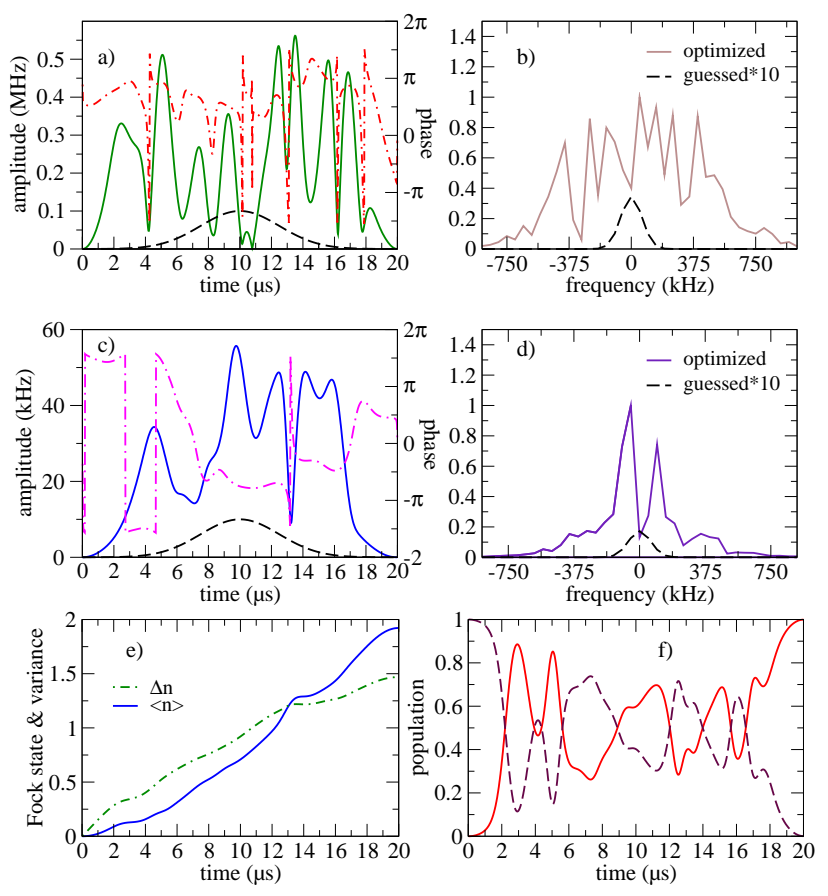

FIG. 7: Preparation of the even cat state with $\alpha=1+\mathrm{i}$ in $\tau=20 \mu$ s using OCT: Temporal dependence of the optimized pulses driving the atom (a) and the cavity (c). The solid and dashed-dotted line correspond to the amplitude and the phase of the fields, respectively (the dashed line shows the guesspulse amplitude amplified by a factor 10, which is a Gaussian pulse with $E_{0}=10 \mathrm{kHz}$ and $\sigma=2.5 \mu \mathrm{s}$ for the atom and with $E_{0}=1 \mathrm{kHz}$ and $\sigma=2.5 \mu$ s for the cavity. Subplots (b) and (d) display the corresponding spectra. The other subplots show the temporal dependence of (e) mean intracavity-photon number $\langle n\rangle$ and standard deviation $\Delta n$ and (f) population of the atomic states $\rho_{g g}$ (solid line) and $\rho_{e e}$ (dashed line). The initial state is $|e, 0\rangle$, the other parameters are given in the text.

also known in the literature as "even cat states" since their decomposition in the Fock state basis only contains even number states. We target $\alpha=1+\mathrm{i}$, which is sufficient for a proof-of-principle discussion. Realizing larger values of $|\alpha|$, in fact, requires a significantly larger numerical effort since the Hilbert space of the cavity has to be truncated at much larger Fock state number $n$. Unlike for the previous classes of states, efficient preparation for the considered time scale requires a pulse that directly drives the resonator. This condition can intuitively be understood since a cavity pump efficiently generates coherent states of the oscillator. For the results reported in this section, the dynamics employs both a pulse driving the resonator, $\tilde{\eta}(t)$, and a pulse which couples to the atomic transition, $\tilde{\Omega}(t)$.

Figure 7 displays the resulting pulses obtained using OCT taking the initial state $|e, 0\rangle$ and the interaction time $\tau=20 \mu \mathrm{s}$. The final infidelity here amounts to $6 \times 10^{-4}$. We observe that the preparation of the even cat state with $\alpha=1+\mathrm{i}$ requires complex pulses, both 
for atom (Fig. 7(a)) and cavity (Fig. 7(b)). Moreover, the amplitude of the atom pump pulse is about one order of magnitude larger than the vacuum Rabi splitting (which scales with $g$ ) and than the amplitude of the cavity pump pulse. This suggests that the field $\tilde{\Omega}(t)$ dresses the atomic levels, whereas the cavity pulse drives selectively the resonances of the dressed atom. This intuition is corroborated by the spectrum of the pulse driving the cavity, shown in Fig. 77(d), where two prominent peaks appear at the transition frequencies between the dressed states. Further information is extracted from the mean photon number in Fig. 7(e) and corresponding variance. They show a steady increase, as it would correspond to a displacement of a harmonic oscillator whose amplitude increases with time until the target value is reached. The atomic dynamics, Fig. 7(f), exhibits oscillations on the time scale of a few microseconds. This is consistent with the picture of the atom pulse dressing the atomic transition. These features thus suggest that the system undergoes a conditional dynamics, such that the cavity pulse drives the two resonances of the dressed atom. This dynamics is reminiscent of proposals for the preparation of Schrödinger cat states based on projective measurements [56], in which even or odd cat states are prepared by means of a conditional dynamics, which realizes a displacement of the oscillator whose sign depends on the internal state of the dressed atom. Differing from these proposals, the preparation presented here is deterministic and thus does not rely on a final projective measurement.

We now analyze the efficiency of preparing an even cat state with $\alpha=1+\mathrm{i}$ for an interaction time of $\tau=10 \mathrm{~ms}$ using OCT. The atom and cavity pulses turn out to be complex. Differing from the previous case, however, their amplitudes are now comparable, and three orders of magnitude smaller than the atom pulse in Fig. 7(a). Figure 8 displays the spectra of atom and cavity pulse for a final infidelity of $8 \times 10^{-5}$. The spectra show well defined resonances at the dressed states of the Jaynes-Cumming dynamics, where the number of states addressed by the fields is now significantly larger. This is consistent with the fact that the ideal cat state corresponds to an infinite sum over the Fock number states. A striking feature is the relative weight between the spectral lines of the atom and the cavity pulse: The latter has a few predominant contributions at low frequencies, corresponding to the lowest dressed states of the ladder. These features seem to suggest that the cavity pump drives selectively and coherently the individual dressed state such that the resulting superposition delivers the target state (16).

Interestingly, the number of OCT iterations required to reach a certain fidelity is independent of the operation time for cat states. This is strikingly different from the preparation of Fock states and Fock state superpositions where time appears to be a resource for control. We attribute this difference to the fact that an infinite sum over Fock states is required for an ideal cat state which cannot perfectly be achieved in either one of the interaction times employed in our optimizations.
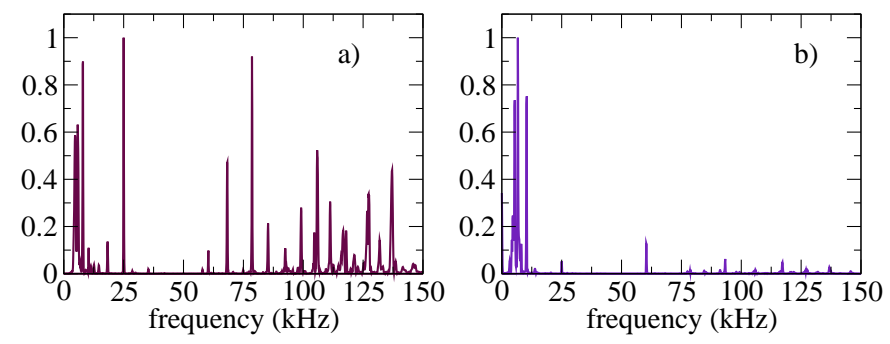

FIG. 8: Preparation of the even cat state with $\alpha=1+\mathrm{i}$ in $\tau=10 \mathrm{~ms}$ using OCT: Positive-frequency spectra of the pulse driving the atom (a) and the cavity (b). The amplitude is in arbitrary units. The initial state is $|e, 0\rangle$, the other parameters are given in the text.

\section{ACCOUNTING FOR EXPERIMENTAL UNCERTAINTIES AND NOISE}

The discussion so far has been concerned with the preparation of high-fidelity quantum states for precisely known interaction times: The pulses which we have determined lead to efficient dynamics as long as the initial and final time of the interaction are well defined. Moreover, we have assumed the parameters of Hamiltonian (5a) to be precisely known.

In this section we investigate the efficiency of the state preparation when taking parameter fluctuations and other sources of uncertainty into account during the optimization. For this purpose we consider the specific situation of the experiment at ENS [21, 22]. We systematically include in our optimization procedure the following experimental features that might compromise our control protocols: (i) the position-dependent coupling of the atom with the cavity mode. In particular, the parameter $g$ in Hamiltonian (2) is replaced by the positiondependent function $g \rightarrow g(x, y, z)$, where $x$ is the propagation direction of the atom with constant velocity $v$, $x=v t$, and $y, z$ are the transverse axes. The functional form reads

$$
g(x, y, z)=g_{0} \mathrm{e}^{-\frac{\left(x-x_{0}\right)^{2}+\left(y-y_{0}\right)^{2}}{\sigma^{2}}} \cos \left(2 \pi\left(z-z_{0}\right) / \lambda\right),
$$

where $x_{0}$ and $y_{0}$ refer to the cavity center in the transverse plane, $z_{0}$ to the closest antinode, $\lambda=5.87 \mathrm{~mm}$ is the cavity-mode wavelength, $\sigma=6 \mathrm{~mm}$ is the mode waist at the cavity center, and $g_{0}=2 \pi \times 50 \mathrm{kHz}$. We further account for the fact that (ii) the atom can only be localized up to a precision of $1 \mathrm{~mm}$ in each direction. This gives rise to a fluctuation in the value of $g$ as well as to fluctuations of the initial and final times of the interaction. In addition, due to the geometric properties of the present cavity-QED setup, (iii) a transverse pulse, $\Omega(t)$, can also directly couple to the cavity mode. Roughly, a $\pi$-pulse on the atom pumps about 20 photons into the cavity mode. Moreover, (iv) temporal fluctuations of the cavity mode frequency occur which can vary up to $\pm 5 \mathrm{kHz}$ in a day. Finally, (v) technical problems in the generation of the 
pulses, such as digitalization and finite time response of the pulse generator, need to be considered.

In our analysis of the optimized solutions' performance we choose the interaction time to be $\tau=\sigma / v \sim 100 \mu \mathrm{s}$ when taking these effects into account. This corresponds to a minimal velocity of the atoms of about $v \sim 60 \mathrm{~m} / \mathrm{s}$. We set the threshold for efficient state preparation by requiring infidelities of the order of $10^{-2}$. This corresponds to the present state-of-the-art of the experimental quantum-state discrimination [44].

Our optimization strategy accounts for these effects by optimizing an ensemble of trajectories whose dynamics are governed by Hamiltonians with different system parameters, but which experience the same control fields [57 59]. Effects (i) and (ii) are implemented by varying the position-dependent coupling $g(x, y, z)$. Specifically, $g(x, y, z)$ is evaluated for three values of $x$, $y$ and $z$, namely, $x=x_{0}, x_{0} \pm 0.5 \mathrm{~mm}$, and analogously for $y$ and $z$. Effect (iii) is simulated by the additional Hamiltonian term

$$
H^{\prime \prime}(t)=\hbar \xi \frac{\Omega(t)}{2}\left(e^{-i\left(\phi_{l}(t)+\omega_{l} t\right)} a^{\dagger}+\text { H.c. }\right),
$$

where $\xi$ is a geometric coefficient deduced from the experiment, which can take a value between 1 and 4 . We consider the worst case and fix the coefficient to take mean value $\xi=4$, with a variation of $\pm 10 \%$. Such a precision in determining $\xi$ could be accessible in an accurate auxiliary measurement using a direct counting of the photon number in the cavity [60]. The variation in the cavity frequency, effect (iv), is accounted for by varying the corresponding parameter in Eq. (5a): specifically, we consider the three values $\omega_{f}=\omega_{f, 0}, \omega_{f, 0} \pm 2 \pi \times 5 \mathrm{kHz}$. Optimization then consists in propagating the same initial state with different Hamiltonians, in order to identify the control fields which yield the best average fidelity. A different approach is required for simulating the effect of digitalization and finite-time response, effect (v). The effect of digitalization is accounted for by fixing the timestep size for changes in the field to $100 \mathrm{~ns}$. Additionally, we model the imprecision and the response of the pulse generator by adding white noise to the atom pulse, with a maximal amplitude of $2 \pi \times 1 \mathrm{kHz}$. Two realizations of the random noise are considered during the optimization. The resulting ensemble comprises 324 system copies when all effects are taken into account. The average fidelity is determined by integrating over the complete parameter ranges.

We first check how much these effects deteriorate the fidelities when not taken into account during the optimization. We consider the target state in Eq. (22), and determine the infidelity for the initial state $(|g\rangle-\mathrm{i}|e\rangle) \otimes$ $|0\rangle / \sqrt{2}$. While the infidelity for the preparation of the target state under ideal conditions is $1.0 \times 10^{-4}$, with the same optimized pulse the infidelity is increased to $9.97 \times 10^{-1}$ when the experimental imperfections are accounted for. It is thus imperative to include the imperfections in the optimization if one is to provide pulses

\begin{tabular}{|l|c|c|c|}
\hline noise effect & $\begin{array}{c}\text { average } \\
\text { optimization } \\
\text { infidelity }\end{array}$ & $\begin{array}{c}\text { size of } \\
\text { optimization } \\
\text { ensemble }\end{array}$ & $\begin{array}{c}\text { average } \\
\text { integrated } \\
\text { infidelity }\end{array}$ \\
\hline (i)-(ii) & $5.0 \times 10^{-3}$ & 27 & $4.6 \times 10^{-3}$ \\
(iii) & $2.2 \times 10^{-2}$ & 2 & $1.3 \times 10^{-2}$ \\
(iv) & $2.1 \times 10^{-4}$ & 3 & $1.6 \times 10^{-4}$ \\
(v) & $6.2 \times 10^{-5}$ & 2 & $1.2 \times 10^{-4}$ \\
\hline
\end{tabular}

TABLE I: Classification of noise impact: (i)-(ii) uncertainty in atom-cavity coupling $g$, (iii) undesired cavity excitation due to atom pulse, (iv) uncertainty of cavity mode frequency, (v) amplitude noise due to digitalization and finite time response of pulse generator. Average optimization fidelity refers to the final value of $J_{\tau}$ evaluated for the system copies in the optimization ensemble, whereas integrated average fidelity corresponds to an integral over the parameter range. The single contributions are to be compared to an average optimization infidelity of $1.7 \times 10^{-1}$ (for an ensemble of 324 system copies) and an average integrated fidelity of $1.6 \times 10^{-1}$ when all noise sources are accounted for simultaneously.

that will be meaningful in the experiment.

When all experimental uncertainties and noise sources are accounted for, using ensemble OCT, the optimized pulses show an improvement by an order of magnitude, leading to an infidelity of about $1.6 \times 10^{-1}$. More importantly, optimization in the presence of noise and imperfections also allows us to identify whether this infidelity is caused by several factors simultaneously or whether it is mainly due to a single source. This is analyzed in Table which reports the individual contributions of each noise effect to the total infidelity of $1.6 \times 10^{-1}$. The optimizations were taken to be converged when $J_{\tau}$ changed by $10^{-6}$ or less. The fact that the largest infidelity of a single noise effect in Table \is about one order of magnitude smaller than the infidelity when accounting for all effects simultaneously, $1.3 \times 10^{-2}$ for effect (iii) compared to $1.6 \times 10^{-1}$, suggests that there is an interplay between two or more of the noise effects that increases the difficulty of the optimization problem substantially. The largest impact on the fidelity, as clearly revealed by inspection of Table प is due to undesired excitation of the cavity by the atom pump pulse. Indeed, we have tested that efficient preparation of Fock state superpositions is achieved by pulses which drive the atom from the side, while large pulses on the cavity field tend to decrease the efficiency over short times. Additionally, in this specific situation, the cavity pulse is proportional to the pulse of the atom, and its effect thus cannot simply be suppressed by better optimization of the atom pulse. We have verified that the fidelity is partially improved by taking a larger value of the vacuum Rabi frequency, $g_{0}=2 \pi \times 100 \mathrm{kHz}$, even for shorter time scales and $\tau=50 \mu \mathrm{s}$. More could be achieved by applying a pulse on the cavity field whose role is to suppress the detrimental effect of Eq. (24). A simpler possibility would be to reduce the parasitic coupling, for instance by proper engineering of the microwave field map. 
In the current work, we have not considered systematic errors in the control parameters $\Omega(t)$ and $\eta(t)$. They may be due to our imperfect knowledge of the real field amplitudes coupled to the atom and the cavity. In contrast to noise, systematic errors may have cumulative effects and lead to faster deterioration of the fidelity of state preparation. Taking them into account in OCT might give preference to solutions featuring adiabatic passage elements instead of resonant population transfers. In this case it is expected that efficient optimization shall resort to the control parameter $\Delta(t)$. We plan to continue the study of this subject in more detail.

\section{CONCLUSIONS}

We have employed optimal control theory to identify dynamics that lead to efficient quantum state preparation of a harmonic oscillator, using a single atom as ancillary quantum system. The pulses which were optimized pump either the atomic transition or the cavity field, or both. The temporal shape of their amplitudes and phases have been determined by optimization using Krotov's method. We have focused on the efficient preparation of three classes of quantum states which have previously been discussed in the literature. This choice has enabled us to compare our results to the dynamics constructed from a physical understanding based on the quantum optical master equations. Our optimized pulses for the preparation of Fock states, Fock state superpositions and Schrödinger cat states yield errors below $10^{-4}$, provided that all parameters of the Hamiltonian are precisely known. Optimal control theory has also allowed us to determine the minimum interaction time required for the quantum state preparation. As expected, it is determined by the atom-cavity interaction strength and the type of state, scaling for example linearly with $n$ for a superposition of the type $(|0\rangle+|n\rangle) / \sqrt{2}$.

We have furthermore evaluated the efficiency of optimal-control-theory-based protocols for prospective applications by taking into account the specific experimental conditions of the setup at ENS. Our analysis shows that it is crucial to include parameter uncertainties and fluctuations in the model. When the noise sources are accounted for during the optimization, optimal control theory can counteract their detrimental influence. This typically improves the error by at least one order of magnitude. Even more importantly, optimal control theory also allows for identifying the noise source with the largest detrimental impact. When experimental imperfections are properly taken into account, optimal control theory can provide an efficient route to quantum-state preparation of arbitrary states. The identified protocols solely rely on deterministic dynamics and are indeed efficient over realistic time scales and for state-of-the-art experimental conditions.

Our protocols can be straightforwardly applied to other physical systems with similar features, for instance, for quantum-state preparation in circuit $\mathrm{QED}$, of the quantized motion of a trapped atom or ion, and of the quantized vibrations of a micromechanical resonator. The robustness against parameter fluctuations has to be calibrated to the specific experiments, but the perspectives are similarly promising to the ones found for microwave cavity QED.

We finally observe that the dynamics implemented here require an efficient determination of the initial state, which has to be uniquely defined. One could also relax this condition and consider implementations which merge optimal control theory with concepts like quantum reservoir engineering $61 \quad 64]$ to realize robust quantum-state preparation [65].

\section{Acknowledgments}

We are grateful to Michel Brune, Daniel Egger, Stefan Gerlich, and in particular to Dietrich Leibfried and Frank Wilhelm, for discussions and helpful comments. Financial support from the German Federal Ministry of Education and Research (BMBF, project QuOReP 16BQ1011), by the European Commission (IP AQUTE), and by the German Research Foundation (DFG) is acknowledged. K. R. acknowledges supports from the StudienStiftungSaar and the kind hospitality of the microwave cavity QED group at ENS in Paris. G.M. thanks the Ion Storage Group at NIST (Boulder) for hospitality during completion of this work.
[1] J. Somlói, V. A. Kazakovski, and D. J. Tannor, Chem. Phys. 172, 85 (1993).

[2] W. Zhu, J. Botina, and H. Rabitz, J. Chem. Phys. 108, 1953 (1998).

[3] J. P. Palao and R. Kosloff, Phys. Rev. Lett. 89, 188301 (2002).

[4] C. Tesch and R. de Vivie-Riedle, Phys. Rev. Lett. 89, 157901 (2002).

[5] J. P. Palao and R. Kosloff, Phys. Rev. A 68, 062308 (2003).

[6] G. De Chiara, T. Calarco, M. Anderlini, S. Montangero,
P. J. Lee, B. L. Brown, W. D. Phillips, and J. V. Porto, Phys. Rev. A 77, 052333 (2008).

[7] T. Caneva, M. Murphy, T. Calarco, R. Fazio, S. Montangero, V. Giovannetti, and G. E. Santoro, Phys. Rev. Lett. 103, 240501 (2009).

[8] M. Murphy, S. Montangero, V. Giovannetti, and T. Calarco, Phys. Rev. A 82, 022318 (2010).

[9] M. H. Goerz, T. Calarco, and C. P. Koch, J. Phys. B 44, 154011 (2011).

[10] A. Konnov and V. Krotov, Automation and Remote Control 60, 1427 (1999). 
[11] S. E. Sklarz and D. J. Tannor, Phys. Rev. A 66, 053619 (2002).

[12] D. M. Reich, M. Ndong, and C. P. Koch, J. Chem. Phys. 136, 104103 (2012).

[13] E. Jaynes and F. Cummings, Proc. IEEE 51, 89 (1963).

[14] C. Law and J. Eberly, Phys. Rev. Lett. 76, 1055 (1996).

[15] A. M. Bloch, R. W. Brockett, and C. Rangan, IEEE Transactions on Automatic Control 55, 1797 (2010).

[16] H. Yuan and S. Lloyd, Phys. Rev. A 75, 052331 (2007).

[17] A. Ben-Kish, B. DeMarco, V. Meyer, M. Rowe, J. Britton, W. M. Itano, B. M. Jelenković, C. Langer, D. Leibfried, T. Rosenband, et al., Phys. Rev. Lett. 90, 037902 (2003).

[18] C. Rangan, A. M. Bloch, C. Monroe, and P. H. Bucksbaum, Phys. Rev. Lett. 92, 113004 (2004).

[19] M. Hofheinz, E. M. Weig, M. Ansmann, R. C. Bialczak, E. Lucero, M. Neeley, A. D. O'Connell, H. Wang, J. M. Martinis, and A. N. Cleland, Nature 454, 310 (2008).

[20] M. Hofheinz, H. W. nd M. Ansmann, R. Bialczak, E. Lucero, M. Neeley, A. O'Connell, D. Sank, J. Wenner, J. Martinis, and A. Cleland, Nature 459, 546 (2009).

[21] J. M. Raimond, M. Brune, and S. Haroche, Rev. Mod. Phys. 73, 565 (2001)

[22] S. Haroche and J. M. Raimond, Exploring the Quantum: Atoms, Cavities, and Photons (Oxford Univ. Press, Oxford, 2006).

[23] B. Mischuck and K. Mølmer, Phys. Rev. A 87, 022341 (2013).

[24] H. Hogben, M. Krzystyniak, G. Charnock, P. Hore, and I. Kuprov, J. Magn Reson. 208, 179 (2011), ISSN 10907807.

[25] Spinach library, http://spindynamics.org/Spinach.php.

[26] L. Davidovich, M. Brune, J. M. Raimond, and S. Haroche, Phys. Rev. A 53, 1295 (1996).

[27] C. Monroe, D. M. Meekhof, B. E. King, and D. J. Wineland, Science 272, 1131 (1996).

[28] D. Leibfried, D. M. Meekhof, B. E. King, C. Monroe, W. M. Itano, and D. J. Wineland, Phys. Rev. Lett. 77, 4281 (1996).

[29] B. Kneer and C. K. Law, Phys. Rev. A 57, 2096 (1998).

[30] P. Domokos, M. Brune, J. Raimond, and S. Haroche, Eur. Phys. J. D 1, 1 (1998).

[31] H. Walther, B. T. H. Varcoe, B.-G. Englert, and T. Becker, Rep. Prog. Phys. 69, 1325 (2006).

[32] P. Filipowicz, J. Javanainen, and P. Meystre, Phys. Rev. A 34, 3077 (1986).

[33] J. J. Slosser, P. Meystre, and S. L. Braunstein, Phys. Rev. Lett. 63, 934 (1989).

[34] M. França Santos, E. Solano, and R. L. de Matos Filho, Phys. Rev. Lett. 87, 093601 (2001).

[35] K. M. Birnbaum, A. Boca, R. Miller, A. D. Boozer, T. E. Northup, and H. J. Kimble, Nature 436, 87 (2005).

[36] A. Kubanek, A. Ourjoumtsev, I. Schuster, M. Koch, P. W. H. Pinkse, K. Murr, and G. Rempe, Phys. Rev. Lett. 101, 203602 (2008).

[37] M. Pechal, C. Eichler, S. Zeytinoglu, S. Berger, A. Wallraff, and S. Filipp, arXiv:1308.4094 (2014).

[38] G. Rempe, F. Schmidt-Kaler, and H. Walther, Phys. Rev. Lett. 64, 2783 (1990).

[39] M. Brune, F. Schmidt-Kaler, A. Maali, J. Dreyer, E. Hagley, J. M. Raimond, and S. Haroche, Phys. Rev. Lett. 76, 1800 (1996).
[40] P. Bertet, S. Osnaghi, P. Milman, A. Auffeves, P. Maioli, M. Brune, J. M. Raimond, and S. Haroche, Phys. Rev. Lett. 88, 143601 (2002).

[41] G. Nogues, A. Rauschenbeutel, S. Osnaghi, M. Brune, J. Raimond, and S. Haroche, Nature 400, 239 (1999).

[42] M. Weidinger, B. T. H. Varcoe, R. Heerlein, and H. Walther, Phys. Rev. Lett. 82, 3795 (1999).

[43] B. T. H. Varcoe, S. Brattke, M. Weidinger, and H. Walther, Nature 403, 743 (2000).

[44] C. Sayrin, I. Dotsenko, X. Zhou, B. Peaudecerf, T. Rybarczyk, S. Gleyzes, P. Rouchon, M. Mirrahimi, H. Amini, M. Brune, et al., Nature 477, 73 (2011).

[45] R. Miller, T. E. Northup, K. M. Birnbaum, A. Boca, A. D. Boozer, and H. J. Kimble, J. Phys. B 38, S551 (2005).

[46] M. Keller, B. Lange, K. Hayasaka, W. Lange, and H. Walther, Nature 431, 1075 (2004).

[47] J. McKeever, A. Boca, A. D. Boozer, R. Miller, J. R. Buck, A. Kuzmich, and H. J. Kimble, Science 303, 1992 (2004).

[48] M. Hijlkema, B. Weber, H. P. Specht, S. C. Webster, A. Kuhn, and G. Rempe, Nature Phys. 3, 253 (2007).

[49] S. T. Merkel and F. K. Wilhelm, New J. Phys. 12, 093036 (2010).

[50] M. Brune, E. Hagley, J. Dreyer, X. Maitre, A. Maali, C. Wunderlich, J. M. Raimond, and S. Haroche, Phys. Rev. Lett. 77, 4887 (1996).

[51] P. Bertet, S. Osnaghi, A. Rauschenbeutel, G. Nogues, A. Auffeves, M. Brune, J. M. Raimond, and S. Haroche, Nature 411, 166 (2001).

[52] C. Hempel, B. P. Lanyon, P. Jurcevic, R. Gerritsma, R. Blatt, and C. F. Roos, Nature Photonics 7, 630 (2013).

[53] V. Giovannetti, S. Lloyd, and L. Maccone, Phys. Rev. A 67, 052109 (2003).

[54] P. Rebentrost, I. Serban, T. Schulte-Herbrüggen, and F. K. Wilhelm, Phys. Rev. Lett. 120, 090401 (2009).

[55] D. J. Egger and F. K. Wilhelm, Supercond. Sci. Technol. 27, 014001 (2014).

[56] E. Solano, G. S. Agarwal, and H. Walther, Phys. Rev. Lett. 90, 027903 (2003).

[57] M. H. Goerz, E. J. Halperin, J. M. Aytac, C. P. Koch, and K. B. Whaley, arXiv:1401.1858 (2014).

[58] K. Kobzar, T. E. Skinner, N. Khaneja, S. J. Glaser, and B. Luy, J. Magn. Reson. 170, 236 (2004), ISSN 10907807.

[59] K. Kobzar, T. E. Skinner, N. Khaneja, S. J. Glaser, and B. Luy, J. Magn Reson. 194, 58 (2008), ISSN 1090-7807.

[60] C. Guerlin, J. Bernu, S. Deléglise, C. Sayrin, S. Gleyzes, S. Kuhr, M. Brune, J. M. Raimond, and S. Haroche, Nature 448, 889 (2007).

[61] J. F. Poyatos, J. I. Cirac, and P. Zoller, Phys. Rev. Lett. 77, 4728 (1996).

[62] A. R. R. Carvalho, P. Milman, R. L. de Matos Filho, and L. Davidovich, Phys. Rev. Lett. 86, 4988 (2001).

[63] C. J. Myatt, B. E. King, Q. A. Turchette, C. A. Sackett, D. Kielpinski, W. M. Itano, C. Monroe, and D. J. Wineland, Nature 403, 269 (2000).

[64] C. Arenz, C. Cormick, D. Vitali, and G. Morigi, J. Phys. B: At. Mol. Opt. Phys. 46, 224001 (2013).

[65] D. M. Reich and C. P. Koch, New J. Phys. 15, 125028 (2013). 